\title{
Detection of microcalcification clusters by 2D-mammography and narrow and wide angle digital breast tomosynthesis
}

\author{
Andria Hadjipanteli ${ }^{\mathrm{a}}$, Premkumar Elangovan ${ }^{\mathrm{b}}$, Padraig T Looney ${ }^{\mathrm{a}}$, Alistair Mackenzie ${ }^{\mathrm{a}}$, Kevin \\ Wells ${ }^{\mathrm{b}}$, David R Dance ${ }^{\mathrm{a}, \mathrm{c}}$, Kenneth C Young ${ }^{\mathrm{a}, \mathrm{c}}$ \\ ${ }^{a}$ National Co-ordinating Centre for the Physics of Mammography, Royal Surrey County Hospital, \\ Guildford, Surrey, GU2 7XX, UK \\ ${ }^{\mathrm{b}}$ Centre for Vision, Speech and Signal Processing, University of Surrey, Guildford, GU2 7XH, UK \\ ${ }^{c}$ Department of Physics, University of Surrey, Guildford, GU2 7XX, UK
}

\begin{abstract}
The aim of this study was to compare the detection of microcalcification clusters by human observers in breast images using 2D-mammography and narrow $\left(15 \% 15\right.$ projections) and wide $\left(50^{\circ} / 25\right.$ projections $)$ angle digital breast tomosynthesis (DBT). Simulated microcalcification clusters with a range of microcalcification diameters $(125 \mu \mathrm{m}-275$ $\mu \mathrm{m})$ were inserted into $6 \mathrm{~cm}$ thick simulated compressed breasts. Breast images were produced with and without inserted microcalcification clusters using a set of image modelling tools, which were developed to represent clinical imaging by mammography and tomosynthesis. Commercially available software was used for image processing and image reconstruction. The images were then used in a series of 4-alternative forced choice (4AFC) human observer experiments conducted for signal detection with the microcalcification clusters as targets. The minimum detectable calcification diameter was found for each imaging modality: (i) $2 \mathrm{D}$-mammography: $164 \pm 5 \mu \mathrm{m}$ (ii) narrow angle DBT: $210 \pm 5 \mu \mathrm{m}$, (iii) wide angle DBT: $255 \pm 4 \mu \mathrm{m}$. A statistically significant difference was found between the minimum detectable calcification diameters that can be detected by the three imaging modalities. Furthermore, it was found that there was not a statistically significant difference between the results of the five observers that participated in this study. In conclusion, this study presents a method that quantifies the threshold diameter required for microcalcification detection, using high resolution, realistic images with observers, for the comparison of DBT geometries with 2D-mammography. 2Dmammography can visualise smaller detail diameter than both DBT imaging modalities and narrow-angle DBT can visualise a smaller detail diameter than wide-angle DBT.
\end{abstract}

Keywords: mammography, digital breast tomosynthesis, microcalcification, breast cancer

\section{INTRODUCTION}

Digital breast tomosynthesis (DBT) is a tomographic technique, which involves the acquisition of two-dimensional projections over a limited angular range and their reconstruction to image planes parallel to the detector. It has been shown that DBT increases the detectability of masses in the breast and reduces recalls when used in combination with 2D-mammography in screening [1]. Some studies have shown that detectability of microcalcifications with DBT is slightly lower than with 2D-mammography, whereas others have claimed the converse is true [2-3]. Therefore, it is still unclear whether the detectability of microcalcification clusters in DBT can be as high as that in 2D-mammography.

The detectability of microcalcifications using DBT is expected to be dependent on the image acquisition methods (e.g. tomographic scan angle, number of projections), the radiation dose used, and the processing and reconstruction methods. All the above factors can limit the detection of small objects such as microcalcifications. The optimum combination of the above variables for DBT is still under investigation. With regards to the DBT system geometry, several studies suggest that the smaller the DBT angle the more likely it is to resolve calcification-like objects in the breast [4-6]. There

Medical Imaging 2016: Physics of Medical Imaging, edited by Despina Kontos, Thomas G. Flohr, Joseph Y. Lo, Proc. of SPIE Vol. 9783, 978306 · C 2016 SPIE · CCC code: 1605-7422/16/\$18 · doi: 10.1117/12.2217663 
is still some disagreement between different studies on the optimum angle and optimum number of projections for microcalcification detection, as different systems, methods and calcification detection metrics were used in each study. No study to date has made a quantitative measurement of the threshold diameter required for microcalcification detection, using high resolution, realistic images with observers, for the comparison of DBT geometries with 2Dmammography.

We have developed a method of quantifying the detection of microcalcification clusters in 2D-mammography and in DBT using an observer study. Here, 2D-mammography has been compared to two DBT geometries; narrow $\left(15^{\circ} / 15\right.$ projections) and wide (50\% $/ 25$ projections) angle DBT. Other factors that can affect calcification detection, dose and processing and reconstruction methods were kept constant between the different imaging modalities. Similarly, the thickness and glandularity of the breast, the height of the inserted cluster in the breast and the cluster diameter were kept constant. Threshold microcalcification diameter was used as the detectability measure for each modality. The method described is reproducible and quantifiable and can be applied to different imaging technologies for masses and microcalcifications.

\section{MATERIALS AND METHODS}

\subsection{Simulated breast tissue}

Simulation methods were used to prepare realistic synthetic breast phantoms. These methods are described in detail elsewhere [7]. Briefly, the breast phantoms were produced by combining breast structures, including glandular tissues, Cooper's ligaments and blood vessels, extracted from reconstructed DBT planes of real patient images. The extracted structures were then de-cluttered, de-noised and shuffled together at random orientations and scale factors to produce anatomical volumetric breast phantoms. These had compressed breast thickness $6 \mathrm{~cm}$ and glandularity $17 \%-19 \%$ by volume. The voxel size in the phantoms was $100 \mu \mathrm{m} \times 100 \mu \mathrm{m} \times 100 \mu \mathrm{m}$. Figure 1 shows an example of simulated (a) 2D-mammography, (b) DBT narrow and (c) DBT wide images of a breast tissue phantom calculated using the method described in section 2.3.

(a)

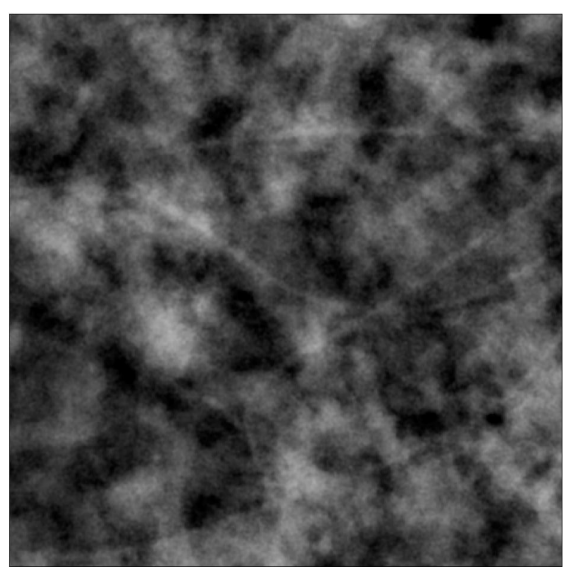

(b)



(c)



Figure 1: Examples of simulated images (cropped to $30 \mathrm{~mm} \times 30 \mathrm{~mm}$ ) of the 3D breast phantom (a) 2D-mammogram, (b) narrow angle DBT plane $30 \mathrm{~mm}$ above the breast support and (c) wide angle DBT plane $30 \mathrm{~mm}$ above the breast support. 


\subsection{Simulated clusters}

Simulated volumes of clusters composed of five microcalcifications were produced. The detection of five microcalcifications in a cluster was regarded as a more realistic representation of the clinical task than detecting a single microcalcification. One high resolution microcalcification image volume was chosen from a database of 400 real microcalcification image volumes, which were acquired using a microcomputed tomography system to image breast biopsy samples [9]. The selected microcalcification was chosen due to its approximately round shape. To form a simulated cluster, the microcalcification was replicated five times with the same diameter, but rotated at a different angle and placed within a $2.5 \times 2.5 \times 2.5 \mathrm{~mm}^{3}$ cubic volume, in such a way that the distance between at least two microcalcifications was $2.5 \mathrm{~mm}$ and there was no overlap of microcalcifications in the planar projection. The above process was repeated by scaling the selected microcalcification to a series of diameters in the range $125 \mu \mathrm{m}$ to $275 \mu \mathrm{m}$. For each microcalcification diameter sixty clusters were produced. The microcalcification clusters were inserted into the breast phantoms at a height of $30 \mathrm{~mm}$ above the breast support table. Figure 2 shows examples of the microcalcification clusters produced with two microcalcification diameters: (a) $125 \mu \mathrm{m}$ and (b) $250 \mu \mathrm{m}$.

(a)

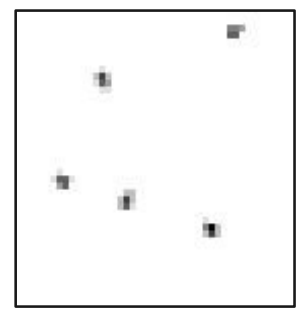

(b)



Figure 2: $2 \mathrm{D}$ projection images of $\left(2.5 \times 2.5 \times 2.5 \mathrm{~mm}^{3}\right.$ cubic volume $)$ clusters with two different microcalcification diameters before insertion: (a) $125 \mu \mathrm{m}$ and (b) $250 \mu \mathrm{m}$.

\subsection{Image simulation}

A set of image modelling tools that simulate clinical imaging by 2D-mammography and DBT were used together with the breast and cluster phantoms described above to produce breast images with and without inserted microcalcification clusters. The detector simulated was amorphous selenium for both 2D-mammography and DBT. The narrow angle DBT geometry tested was based on a $15 \% 15$ projections configuration. In addition, a wide angle DBT geometry was tested, based on a $50^{\circ} / 25$ projections configuration. The $\mathrm{kVp}$ and target/filter materials used in the $\mathrm{x}$-ray simulation were: (i) 2D: $31 \mathrm{kVp} \mathrm{W} / \mathrm{Rh}$, (ii) DBT: $33 \mathrm{kVp} \mathrm{W} / \mathrm{Al}$, which are typical of those used clinically for a $6 \mathrm{~cm}$ thick breast. The primary projections were produced using a ray-tracing tool [10]. The pixel size in $2 \mathrm{D}$-mammography was $70 \mu \mathrm{m}$ and in both DBT systems it was $140 \mu \mathrm{m}$ (binned before processed by image processing and reconstruction software). Scatter was calculated using Monte Carlo simulations for a $6 \mathrm{~cm}$ thick breast and added to the images. The spectra used were scaled so that the mean glandular dose [11,12] to the breast was in all cases $2.5 \mathrm{mGy}$ [13]. The ray tracing simulation included the effect of the grid transmission factor, tube half value layer, geometric blurring due to the focal spot finite size, tube movement blurring of the focal spot, attenuation in the breast support and compression paddle, as appropriate to the three imaging modalities. No movement of the breast was taken into account in the simulation. Finally, the methods of Mackenzie et al $[14,15]$ were used in the simulation process to further blur and add noise to the images appropriate for the detector and radiation dose being simulated.

Briona (Real Time Tomography, LLC, Philadelphia, USA) software was used for the processing and reconstruction of images. Briona uses a back projection reconstruction method with iterative and non-linear processing techniques to mitigate noise and artifacts. The Briona software was used as it provides flexibility to the user in modelling different DBT geometry configurations. Different DBT geometry configurations were set up for the two DBT imaging modalities. The same reconstruction method and filters were used for the two DBT modalities. Quantitative analysis (full width at half maximum, contrast-to-noise ratio (CNR), contrast degradation factor) and qualitative tests on DBT images 
reconstructed using Briona showed that it produced images comparable to those produced by other reconstruction and processing software used clinically.

After processing and reconstruction, the 2D-mammography images were cropped into $30 \mathrm{~mm} \times 30 \mathrm{~mm}$ images and the DBT planes were cropped to $30 \mathrm{~mm} \times 30 \mathrm{~mm} \times 10 \mathrm{~mm}$ images to be used in 4-alternative forced choice (4AFC) human observer experiments.

\subsection{Observation and 4 AFC study}

The images produced using the above methods were used in a series of 4AFC human observer experiments on the detection of microcalcification clusters. For each modality the threshold diameter for microcalcification detection was determined.

In the 4AFC study sets of four $30 \mathrm{~mm} \times 30 \mathrm{~mm}$ breast phantom $2 \mathrm{D}$ images were randomly selected and shown in turn to the observer. In each set one image contained a microcalcification cluster in the centre and the other three did not. The images were presented using an in-house graphical user interface (GUI) as shown in figure 3 . The location of the image containing the lesion was random. A 2D projection of the inserted 3D cluster was also shown without the background. This was repeated using DBT images where the observer could scroll through 10 planes, and the central planes always contained the centre of the cluster. The initial DBT plane to be displayed to the observer was chosen at random and the DBT stack displayed wrapped around when the observer scrolled to the end of the stack.

Five physicists participated in the 4AFC study. Each observer was shown in total 540 groups of four images (one with cluster and three without): 180 groups of images for each modality, which was composed of three sets of 60 images with a microcalcification, each set with a different microcalcification diameter. The appropriate diameters for each modality were chosen after a pilot study.



Figure 3: Example image set as displayed in the 4AFC GUI with signal cue for the $2 \mathrm{D}$ study containing a $175 \mu \mathrm{m}$ microcalcification cluster. The red arrow has been added to the image to indicate which of the four images contains the cluster. 
The observers had to decide which of the four images contained the cluster and register the decision by selecting the relevant quadrant. All experiments were undertaken on a high-resolution monitor (Barco B-8500, 5MP, Belgium). All images were displayed at $100 \%$ magnification (one to one pixel between the image and the monitor) and no changes in magnification were allowed. Low lighting levels were set and no time limit was imposed. The minimum detectable calcification size was defined as the value at which the observer makes $92.5 \%$ correct decisions for a given experimental condition, which corresponds to a detectability index $d^{\prime}$ of 2.5. A linear relationship between the detail diameter and the detectability index can be assumed, if the experiment is unbiased [16]. The diameter required to achieve a $d^{\prime}$ of 2.5 for each modality and observer was determined by linear least square fitting and the overall mean $d^{\prime}$ for each modality was then calculated. Diameters that resulted in a detection rate of $100 \%$ were not included and in such cases a linear two point fit to the data was used.

Analysis of variance (ANOVA) was performed on the results to identify any statistically significant differences between the minimum detectable calcification diameters of the three different imaging modalities. In addition, ANOVA was performed on the results to investigate the differences between the observers on the minimum detectable calcification diameter.

\section{RESULTS}

Figure 5 shows the detectability index, $d$, versus calcification diameter results for 2D-mammography for a single observer. The increase in detectability index with calcification diameter is shown to follow a linear relationship as expected. Similarly good fits were obtained for all modalities and observers where three data points were available. In four of the 15 modality/observer combinations, the detectability for the largest calcification diameter was $100 \%$ and only two data points were used.

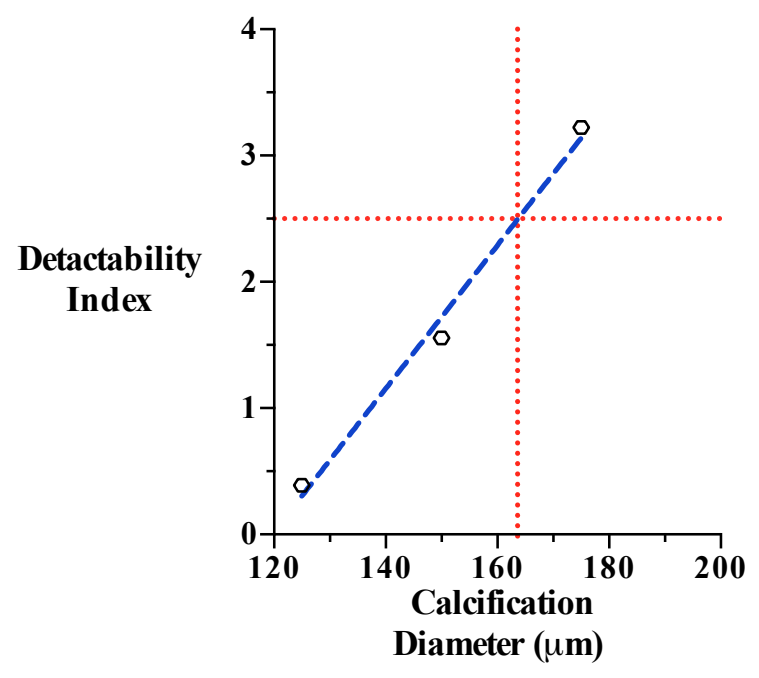

Figure 5: The detectability index, $d$, versus calcification diameter results for 2D-mammography for one of the observers. The calcification diameter at a detectability index of 2.5 was considered the minimum detectable calcification diameter as found by that observer. 
Figure 6 shows the minimum detectable calcification diameter for each imaging modality: (i) 2D-mammography: 164 \pm 5 $\mu \mathrm{m}$ (ii) DBT narrow angle: $210 \pm 5 \mu \mathrm{m}$, (iii) DBT wide angle: $255 \pm 4 \mu \mathrm{m}$. The errors in the minimum detectable calcification diameter were calculated as the standard error in the average minimum detectable calcification diameter from all the observers.

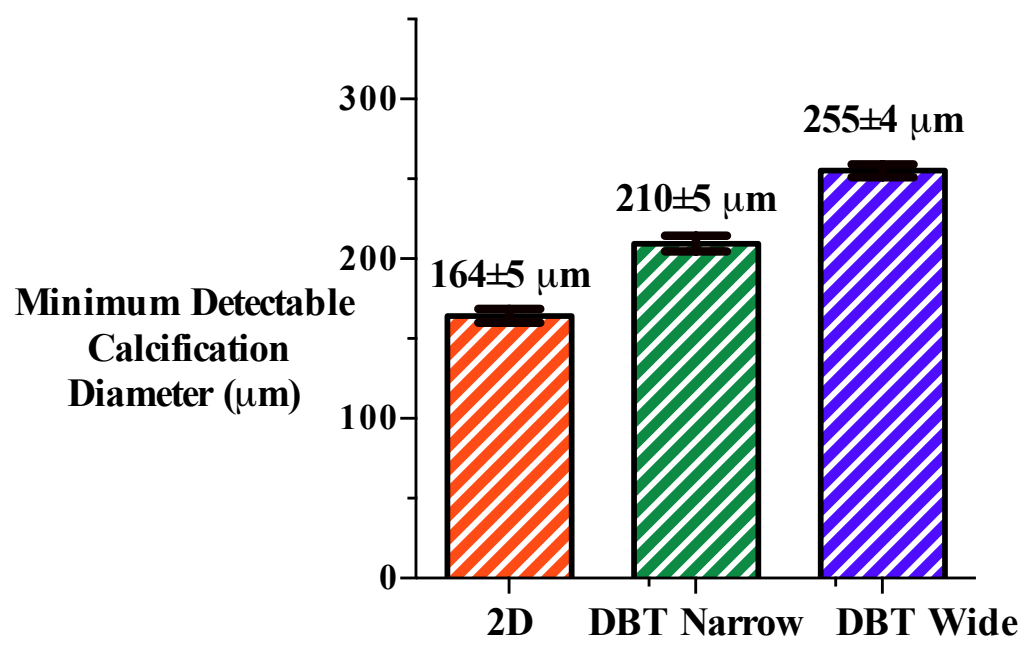

Figure 6: The minimum detectable calcification diameter for each imaging modality.

ANOVA was performed to test if the minimum detectable calcification diameters of the three modalities were significantly different. A $p$-value of $<0.0001$ was found showing that there was a highly significant statistical difference between the minimum calcification diameter that can be detected by 2D-mammography, narrow and wide angle DBT. Furthermore, ANOVA gave a $p$-value of 0.50 for the effect of the variation between the observers, showing confidence in the rejection of the hypothesis that there is significant difference in the calcification diameter found by the five observers that participated in this study.

\section{DISCUSSION}

2D-mammography was found to have a smaller threshold calcification diameter than both DBT imaging modalities and narrow-angle DBT had a smaller threshold calcification diameter than wide-angle DBT. The DBT images are less sharp than the 2D images as the modulation transfer function (MTF) is lower. This is due to the larger pixel pitch of DBT compared to 2D-mammography (due to pixel binning in DBT) and the tube movement in DBT, which introduces blurring. Also, a slight further increase in geometric blurring might be introduced as the angle becomes wider. The reduced MTF may partially explain why 2D-mammography was found to detect smaller calcifications than narrow angle DBT.

At wider angles the path of X-rays through the tissue is greater, so the signal reaching the detector is lower and the relative noise increases. To keep the same MGD between the DBT acquisition modes, the dose per projection needs to be decreased for wide angle DBT, thus the relative noise in each projection is increased. Sechopoulos et al [6] found that for a constant angle, increasing the number of projection decreased the CNR. Also, the total electronic noise in wide angle DBT simulated in this study is higher than the total electronic noise in narrow angle DBT, due to the higher number of projections. The above reasons may explain why narrow angle DBT performs better than wide angle DBT for calcifications. It has to be noted here that a different wide angle DBT system with lower electronic noise might have better performance than the system in this study. 
This study has the advantage of testing the effect of acquisition method on calcification detection using real observers. However, this does limit the number of acquisition methods that can be practicably studied. Other studies [5, 6], which used metrics (e.g. CNR, CNR/ASF (artefact spread function)) instead of observer experiments to assess the visibility of calcifications, tested more geometries but they lacked the link to the detection performance of a real observer. In mammography, a calcification cluster may contain a variable number of calcifications of different sizes and shapes. Here we used a simplified cluster with five identical calcifications spread over a volume. Many clusters contain larger calcifications, which may be more easily detectable and so caution must be taken in applying these results to the detection of all calcification clusters. It was advantageous to use a cluster rather than a single calcification as this better simulates the clinical task.

Finally, an attempt was made to employ conditions and methods that are clinically used which makes this study relevant to existing clinical systems. It has to be stated that the above findings cannot necessarily be generalised, for example, to other doses, acquisition geometries or other processing and reconstruction methods. The methods used here can be extended to study the detectability of masses by different imaging modalities. In a preliminary investigation we found that masses were better detected by narrow DBT than mammography [17].

\section{CONCLUSIONS}

Although previous publications have compared calcification detection between different DBT geometries, none have used a reproducible quantitative approach with observers, calcification clusters and clinically realistic backgrounds to compare different DBT geometries and 2D imaging.

This study has shown that the method described can quantify the detectability of calcification clusters for different breast imaging modalities using observer studies. The results show that small calcifications in clusters can be more reliably detected using 2D mammography than DBT.

\section{ACKNOWLEDGEMENTS}

This work is part of the OPTIMAM2 project (grant number: C30682/A17321) funded and supported by Cancer Research UK. The authors would like to thank their colleagues at the National Co-ordinating Centre for the Physics of Mammography and at the Regional Radiation Protection Service, Royal Surrey County Hospital, for participating in this study. We are also grateful to the staff of Real Time Tomography for their help in using their software in this experiment.

\section{References}

1. Rafferty, E. A., Park, J. M., Philpotts, L. E., Poplack, S. P., Sumkin, J.H. and Niklason, L. T., “Assessing radiologist performance using combined digital mammography and breast tomosynthesis compared with digital mammography alone: results of a multicentre, multireader trial," Radiology 266(1), 104-113 (2013).

2. Spangler, M. L., Zuley, M. L., Smukin, J. H., Abrams, G., Ganott M. A., Hakim, C., Chough, D. M., Shah, R., and Gur, D., "Detection and classification of calcifications on digital breast tomosynthesis and 2D digital mammography: a comparison," AJR Am J Roentgenol 196(2), 320-324 (2011).

3. Kopans, D., Gavenonis, S., Halpern, E., and Moore, R., "Calcifications in the breast and digital breast tomosynthesis," Breast J 17(6), 638-644 (2011).

4. Chan, H. P., Goodsitt, M. M., Helvie, M. A., Zelakiewicz, S., Schitz, A., Noroozian, M., Paramagul, C., Roubidoux, M. A., Nees, A. V., Neal, C. H., Carson, P., Lu, Y., Hadjiski, L. and Wei, J., "Digital breast tomosynthesis: Observer performance of clustered microcalcification detection on breast phantom images acquired with an experimental system using variable scan angles, angular increments and number of projection views," Radiology 273(3), 675-685 (2014).

5. Petersson, H., Dustler, M., Timgberg, A., Timberg, P., "Monte Carlo simulation of breast tomosynthesis: visibility of microcalcifications at different acquisition schemes," Proc. SPIE 9412, 94121H1-94121H7 (2015). 
6. Sechopoulos, I., and Ghetti, C., "Optimization of the acquisition geometry in digital tomosynthesis of the breast,".Med. Phys. 36(4), 1199-1207 (2009).

7. Elangovan, P., Dance, D. R., Young, K. C., and Wells, K., “Simulation of 3D synthetic breast blocks," Proc. SPIE Medical Imaging (accepted 2016).

8. Warren, L. M., Dummott, L., Wallis, M. G., Given-Wilson, R. M., Cooke, J., Dance, D. R. and Young, K. C., "Characterisation of screen detected and simulated calcification clusters in digital mammography," Proc. IWDM 8539, 364-371 (2014).

9. Shaheen, E., Van Ongeval, C., Zanca, F., Cockmartin, L., Marshall, N., Jacobs, J., Young, K. C. and Dance, D. R., "The simulation of 3D microcalcification clusters in 2D digital mammography and breast tomosynthesis," Med. Phys. 38(12), 66596671 (2011).

10. Elangovan, P., Warren, L. M., Mackenzie, A., Diaz, O., Rashidnasab, A., Dance, D. R., Bosmans, H., Young, K. C. and Wells K., "Development and validation of a modelling framework for simulating 2D-mammography and breast tomosynthesis images," Phys. Med. Biol. 59(15), 4275-4293 (2014).

11. Dance, D. R., Skinner C. L., Young, K. C., Beckett, J. R. and Kotre, C. J., "Additional factors for the estimation of mean glandular breast dose using the UK mammography dosimetry protocol," Phys. Med. Biol. 45(11), 3225-3240 (2000).

12. Dance, D. R., Young, K. C. and Engen, R. E., "Estimation of mean glandular dose for breast tomosynthesis: factors for use with the UK, European and IAEA breast dosimetry protocols," Phys. Med. Biol. 56(2), 453-472 (2011).

13. Bouwman, R. W., van Engen, R. E., Young, K. C., den Heeten, G. J., Broeders, M. J., Schopphoven, S., Jeukens, C. R., Veldkamp W. J., and Dance D. R., "Average glandular dose in digital mammography and digital breast tomosynthesis: comparison of phantom and patient data," Phys. Med. Biol. 60(20), 7893-7907 (2015).

14. Mackenzie, A., Dance, D. R., Workman, A., Yip, M., Wells, K. and Young, K. C., "Development and validation of a method for converting images to appear with noise and sharpness characteristics of a different detector and X-ray system," Med. Phys. 39(15), 2721-2734 (2012).

15. Mackenzie, A., Dance, D. R., Diaz, R. and Young, K. C., "Image simulation and a model of noise power spectra across a range of mammographic beam qualities," Med. Phys. 41(12), 121901-1-14 (2014).

16. Burgess, A. E., "Comparison of receiver operating characteristic and forced choice observer performance measurement method," Med. Phys. 22(5), 643-655 (1995).

17. Elangovan, P., Rashidnasab, A., Mackenzie, A., Dance, D. R., Young, K. C., Bosmans, H., Segars, W. P. and Wells, K., "Performance comparison of breast imaging modalities using a 4AFC human observer study," Proc. SPIE 9412 94121T-194121T-7 (2015). 